\title{
30 Anos de Investigação sobre Externalidades do IDE para as Empresas Nacionais - Que Conclusões?
}

\author{
- NUNO CRESPO* \\ - MARIa PAUla Fontoura **
}

\begin{abstract}
RESUMO
Durante as últimas três décadas, surgiu uma vasta literatura sobre a existência potencial de externalidades do IDE para as empresas nacionais, traduzidas em ganhos de produtividade. Esta ocorrência relaciona-se com o fato de as empresas multinacionais possuírem ativos intangíveis que podem ser transmitidos às empresas nacionais. A análise empírica não dá, contudo, indicações claras sobre o fenômeno. Nesta análise, abordamos o mais vasto conjunto de estudos sobre este tema até hoje reunido, levando em consideração a ocorrência de externalidades intra e inter-setoriais. Destacamos a diversidade de canais e respectivos efeitos através dos quais as externalidades para as empresas nacionais se podem materializar. Delineamos ainda rumos para um melhor esclarecimento dos fatores condicionantes da ocorrência do fenômeno, considerando que diversos estudos empíricos têm mostrado que este existirá, provavelmente, apenas para um subgrupo de empresas nacionais e/ou quando estiverem reunidas determinadas condições.
\end{abstract}

\section{Palavras-Chave}

externalidades intra-setoriais, externalidades inter-setoriais, evidência empírica, empresas nacionais, produtividade

\begin{abstract}
During the last three decades a vast literature emerged on the empirical evidence of productivity spillovers from FDI. This is related to the fact that multinational firms own intangible assets which may be transmitted to domestic firms and thereby raise their productivity level. Results obtained so far are however mixed, and it does not seem possible to get a straightforward answer to whether this phenomenon occurs. This paper analyses the most extensive number of studies on the subject surveyed to date, by taking into account intra and inter FDI spillovers. We summarize the different channels for diffusion of FDI spillovers and the effects that may be expected in each case. Some paths for further research on the topic are also delineated, which take into consideration the fact that the existence of the phenomenon appears to depend on the sectoral characteristics and some conditioning factors.
\end{abstract}

\section{KEY WORDS}

intra-sectoral spillovers, inter-sectoral spillovers, empirical evidence, domestic firms, productivity

\section{JEL ClassificAtion}

F2I, F23

* ISCTE. E-mail: nuno.crespo@iscte.pt.

* ISEG - Universidade Técnica de Lisboa. End. para contato: Rua Miguel Lúpi, 20 -1200-78I - Lisboa - Portugal. Email: fontoura@iseg.utl.pt.

(Recebido em janeiro de 2005. Aceito para publicação em março de 2007). 


\section{INTRODUÇÃO}

O Investimento Direto Estrangeiro (IDE) é, atualmente, uma das faces mais visíveis do processo de globalização. Após décadas de ceticismo, «existe hoje uma crença generalizada de que as empresas multinacionais podem ser um elemento importante na estratégia de desenvolvimento de um pais» (Rodríguez-Clare, 1996, p. 852), freqüentemente consubstanciada num tratamento preferencial concedido à empresa multinacional (EM) e na eliminação de diversas barreiras à entrada de IDE. ${ }^{1}$

Os efeitos do IDE nas economias receptoras são, usualmente, diferenciados entre efeitos diretos e indiretos. Os primeiros referem-se, por exemplo, à formação de capital, criação de emprego, aumento das receitas fiscais ou à alteração da estrutura produtiva e exportadora dos países receptores. Porém, a razão principal pela qual os países procuram captar IDE reside, provavelmente, na expectativa de acederem a tecnologia - entendida num sentido lato que contempla não apenas as técnicas de produção ou distribuição como também técnicas de gestão e marketing - mais moderna (Blomström; Kokko, 1998). Estes são os designados efeitos indiretos do IDE. Através de diversos canais, as firmas nacionais podem se beneficiar da superior tecnologia detida pelas EM, obtendo ganhos de produtividade. Se esses ganhos não forem totalmente absorvidos pelas EM, serão geradas externalidades do IDE para as empresas nacionais. Estas externalidades poderão ocorrer quando a empresa estrangeira e a empresa nacional se situam no mesmo setor (externalidades intra-setoriais) e/ou quando pertencem a setores diferentes (externalidades inter-setoriais), como acontece quando as EM estabelecem relações com empresas nacionais fornecedoras ou compradoras de insumos intermediários (respectivamente conhecidas por backward e forward linkages).

A avaliação empírica da existência destes efeitos indiretos do IDE constitui, portanto, um tema de particular interesse para as economias receptoras de IDE, tendo suscitado uma vasta investigação ao longo dos últimos 30 anos, com especial incidência a partir de meados da década de 1990. O objetivo deste trabalho consiste em analisar as respostas que resultam desta investigação. Assim, consideramos o mais amplo conjunto de estudos até hoje reunido para uma investigação desta natureza e evidenciamos a mensagem central que deles pode ser extraída no que se refere à existência deste tipo de benefício para as empresas nacionais decorrente da presença das EM.

O trabalho está estruturado em cinco seções. Após estas observações introdutórias, a segunda seção foca os canais através dos quais as externalidades do IDE se podem concretizar. As seções seguintes abordam a evidência empírica relativa à ocorrência de

1 Ver Hanson (2001), para uma referência às diferentes formas que o apoio à entrada de IDE tem assumido. 
externalidades. A terceira seção analisa as externalidades intra-setoriais (subseção 3.1) e as externalidades inter-setoriais (subseção 3.2). A quarta seção traça algumas vias para o desenvolvimento da investigação sobre este tema. Finalmente, a quinta seção esboça algumas observações conclusivas.

\section{OS CANAIS DE GERAÇÃO DE EXTERNALIDADES}

A literatura que tem investigado este tema evidencia diversos canais de geração de externalidades tecnológicas em benefício das empresas locais. Esses canais de difusão podem ser estruturados em cinco grandes grupos: processo de demonstração/imitação, mobilidade do fator trabalho, exportação, concorrência e relações com fornecedores e compradores locais. Seguidamente, descrevemos a fundamentação subjacente a cada uma destas vias.

O processo de demonstração (por parte das EM)/ imitação (pelas empresas nacionais) é, provavelmente, o canal mais evidente de geração de externalidades (Das, 1987; Wang e Blomström, 1992). A introdução de uma nova tecnologia em um mercado poderá ser dispendiosa e arriscada para uma empresa nacional, pois implica custos de informação sobre a nova tecnologia e acarreta alguma incerteza em relação ao sucesso da sua aplicação no mercado local. Neste contexto, a introdução da tecnologia por parte da EM pode representar uma demonstração de sucesso, incentivando a empresa local a adotá-la. Naturalmente, a relevância deste efeito é acrescida quando o bem produzido pela EM é um substituto próximo daquele que a empresa nacional produz (Barrios e Strobl, 2002).

O segundo canal citado refere-se à possibilidade de as empresas locais contratarem trabalhadores que, tendo trabalhado nas EM, conheçam a tecnologia e possam implementá-la na empresa local, assegurando o seu funcionamento. Fosfuri et al. (2001) e Glass e Saggi (2002) apresentam modelos teóricos compatíveis com esta idéia, sendo a evidência desta mobilidade - mais nítida em países desenvolvidos - exposta, por exemplo, por Gershenberg (1987), Caves (1996) ou Pack (1997). ${ }^{2}$ O fato de as EM tipicamente fornecerem mais formação (Gonçalves, 1986; Gershenberg, 1987; Narula e Marin, 2003) e contribuírem para o enriquecimento do capital humano das economias locais reforça a relevância potencial deste canal como veículo de externalidades para as empresas nacionais. Porém, a influência desta mobilidade do trabalho sobre a eficiência das empresas locais é, como se torna evidente, mais difícil de avaliar, pois implica a necessidade de seguir o percurso dos trabalhadores e averiguar a sua influência na produtividade das empresas nacionais (Saggi, 2002). Não causa surpre-

2 Para uma visão mais detalhada sobre este domínio, ver Blomström e Kokko (2003). 
sa, portanto, a inexistência de estudos detalhados sobre esta vertente. É importante salientar, contudo, um possível impacto negativo resultante do fato de as EM - fruto do superior nível salarial praticado - poderem atrair os melhores trabalhadores das empresas nacionais (Sinami e Meyer, 2004).

Um terceiro canal mediante o qual a presença das EM pode beneficiar as empresas locais é através de exportações (Aitken et al., 1997; Greenaway et al., 2004). Paralelamente à análise da influência das EM na produtividade destas empresas - foco da presente análise -, tem sido desenvolvida uma outra vertente - mais reduzida - que visa investigar o impacto das EM sobre a capacidade exportadora das empresas locais. ${ }^{3}$ Os estudos existentes demonstram que a presença das EM contribui positivamente para a capacidade exportadora das empresas nacionais (Rhee, 1990; Aitken et al., 1997; Kokko et al., 2001). Efetivamente, a atividade de exportação envolve um conjunto de custos associados, por exemplo, ao estabelecimento de redes de distribuição e de transporte, conhecimento das preferências dos consumidores nos mercados exteriores, entre outros aspectos. O fato de as EM, pela sua própria natureza, serem mais aptas a ultrapassar estes fatores, pode facilitar o processo exportador das empresas nacionais, pois estas, através de imitação (ou, em circunstâncias específicas, por colaboração), podem ter um mais amplo conhecimento e minimizar os custos de entrada no mercado externo. Os ganhos assim obtidos poderão repercutir favoravelmente na eficiência produtiva das empresas nacionais. Vários estudos - Bernard e Jensen (1999), Sgard (2001), Girma (2003), Greenaway et al. (2004) - evidenciam o fato de as empresas exportadoras terem níveis superiores de produtividade. ${ }^{4}$ Este resultado pode ser entendido à luz de alguns dos fatores que funcionam no âmbito interno. Assim, é expectável que empresas exportadoras operem, em média, numa escala produtiva superior, o que, existindo rendimentos crescentes, poderá gerar um incremento da produtividade. Por outro lado, a concorrência no mercado externo pode, de igual modo, estimular uma utilização dos recursos mais eficiente e conduzir à adoção de novas tecnologias, como veremos no âmbito do próximo canal considerado nesta seção. Em suma, os acréscimos em termos de capacidade de exportação das empresas nacionais, induzidos pela presença das EM, podem transformar-se em ganhos de eficiência para as primeiras, traduzidos num aumento da sua produtividade.

Um quarto canal de concretização de externalidades está relacionado com o acréscimo de concorrência gerado pela presença das EM (Wang e Blomström, 1992; Markusen e Venables, 1999). No quadro do modelo de Wang e Blomström (1992), as externalidades do IDE dependem não apenas da dimensão da presença externa, mas também da concorrência, na economia nacional, entre a EM e a empresa nacional. A concorrência

3 Uma visão sintética desta vertente de avaliação é apresentada por Görg e Greenaway (2004).

4 Girma (2003), por exemplo, detecta que as empresas exportadoras são, em média, 8,29\% mais produtivas que as não-exportadoras. 
com as EM constitui um incentivo para as empresas locais utilizarem os recursos e a tecnologia existente de modo mais eficiente ou até para a adoção de novas tecnologias, visando enfrentar essa concorrência. Todavia, a presença das EM pode, através deste canal, exercer uma influência negativa sobre a eficiência das empresas locais, pois poderá provocar significativas perdas de cota de mercado, forçando-as a produzir numa escala menos eficiente, com o conseqüente aumento dos seus custos médios (Harrison, 1994; Aitken e Harrison, 1999). Se o efeito for significativo, algumas empresas poderão ser forçadas a sair do mercado. Este efeito não deve, necessariamente, ser encarado negativamente, pois poderá conduzir à saída do mercado de empresas locais menos eficientes, gerando um aumento da produtividade média (Barrios e Strobl, 2002).

Uma última via através da qual as EM podem beneficiar as empresas locais diz respeito às relações que estas estabelecem, no mercado local, com as EM, enquanto suas fornecedoras (backward linkages) ou compradoras de insumos intermediários (forward linkages), como salientado, por exemplo, por Lall (1980) e formalizado em RodríguezClare (1996), Markusen e Venables (1999) ou Lin e Saggi (2004).

Consideremos, em primeiro lugar, as externalidades através de backward linkages. Existindo rendimentos crescentes de escala, a presença das EM pode beneficiar as empresas fornecedoras na medida em que aumenta a procura de insumos locais. $\mathrm{O}$ ganho potencial para as empresas locais poderá, também, envolver uma relação direta com a EM, decorrendo do interesse desta última em assegurar um determinado padrão de qualidade. Nesse caso, a EM poderá auxiliar as empresas fornecedoras em variados aspectos: apoio técnico visando a melhoria da qualidade dos bens produzidos ou a introdução de inovações, por exemplo através da formação de trabalhadores; apoio na criação de infra-estruturas produtivas ou na aquisição de matérias-primas; apoio em termos organizacionais e de gestão, entre outros aspectos (Lall, 1980). ${ }^{5}$ Deve-se também considerar os possíveis benefícios de eficiência para as empresas nacionais induzidos pela concorrência entre elas, já que as empresas têm como objetivo tornarem-se fornecedoras das EM.

As externalidades das EM sobre as empresas locais podem ocorrer também através de forward linkages. ${ }^{6}$ A forma mais evidente de concretização deste canal consiste no fornecimento de insumos de maior qualidade e/ou a um preço inferior às empresas locais produtoras de bens finais (Markusen; Venables, 1999). No entanto, não é possível excluir que o aumento de qualidade na produção produza um aumento do preço e, se as empresas nacionais não tiverem capacidade para se beneficiar com o acréscimo de qualidade nos insumos adquiridos, elas se limitarão a suportar um custo acrescido, gerando-se um efeito negativo através de forward linkages (Javorcik, 2004).

5 Sobre este assunto, ver, ainda, Blalock (2001) e Gonçalves (2005).

6 Apesar disso, por vezes é argumentado que a dimensão deste efeito tenderá a ser menor, dado que as EM concentram grande parte da sua atividade em bens de consumo final (Damijan et al., 2003b). 
Os quatro primeiros mecanismos descritos estão ligados, fundamentalmente, às chamadas externalidades intra-setoriais (ou horizontais), enquanto o último canal se refere a externalidades inter-setoriais (ou verticais). É, contudo, difícil, como salienta Kinoshita (2001), distinguir na prática os diferentes canais, dado que os mecanismos geradores de externalidades do IDE são complexos e interdependentes.

Esta breve sistematização dos canais de suscitação de externalidades para as empresas nacionais evidencia, ainda, a existência de diversos efeitos de sinal contrário, impedindo a criação de uma expectativa clara quanto ao seu impacto global. Deste modo, a sua ocorrência é, fundamentalmente, uma questão de avaliação empírica sobre o que acontece na realidade (Barrios; Strobl, 2002, p. 463).

\section{EXTERNALIDADES DO IDE}

Antes de abordarmos a evidência relativa a esta temática, impõem-se duas notas de natureza metodológica. Em primeiro lugar, sendo nosso objetivo responder, de forma global, à questão relativa à existência de externalidades, recorremos, nos diferentes trabalhos, aos resultados mais abrangentes em termos da amostra utilizada e cujas metodologias (modelo econométrico) são consideradas mais adequadas para lidar com as características particulares das amostras. Em segundo lugar, visando assegurar a compatibilidade entre as conclusões decorrentes dos diferentes estudos, será considerada significativa a influência externa sobre a produtividade das empresas domésticas quando a estatística $t$ associada indicar que a variável é significativa a um nível de $10 \%$.

O procedimento mais comum para avaliar a existência de externalidades do IDE tem consistido numa regressão em que a produtividade (total ou do fator trabalho), o seu crescimento, ou uma outra medida que vise captar a eficiência das empresas nacionais é assumida como variável dependente; como variáveis independentes, são considerados diversos elementos suscetíveis de influenciar essa eficiência, incluindo uma variável que capte a importância relativa da presença estrangeira no setor em causa. São múltiplas as variáveis que têm sido incluídas como fatores determinantes da eficiência nacional. Entre estas, destacam-se - embora nelas não se esgotando - medidas de economias de escala, concentração setorial (concentração de mercado), intensidade de capital e qualificação do trabalho. ${ }^{7}$ Em alguns casos, são adicionadas variáveis que procuram distinguir o canal específico de transmissão das externalidades e o seu respectivo impacto sobre o nível de eficiência das empresas nacionais.

7 Dado o objetivo específico deste trabalho ser o de abordar a existência de externalidades do IDE, a influência destas variáveis não é analisada. 


\subsection{Externalidades Intra-Setoriais do IDE}

As últimas três décadas assistiram à emergência de um vasto conjunto de estudos visando a avaliação da existência de externalidades intra-setoriais cujas conclusões se caracterizam, essencialmente, pela diversidade. Recentemente, tem sido argumentado que uma parte significativa dessa divergência de resultados se deve ao tipo de informação utilizada nos diversos estudos, com destaque para o fato de os trabalhos recorrerem a dados cross-section ou em painel (Görg e Strobl, 2001; Görg e Greenaway, 2004). De fato, tem sido defendido que a utilização de dados em painel e desagregação por empresa/estabelecimento constitui a forma mais adequada para investigar a existência de externalidades do IDE para as empresas nacionais (Görg e Strobl, 2001; Görg e Greenaway, 2004). Por um lado, a principal dificuldade associada à utilização de dados cross-section reside na impossibilidade de se estabelecer o sentido da causalidade. Um sinal positivo do coeficiente que capta a dimensão da presença externa pode significar a existência de externalidades positivas para as empresas nacionais ou espelhar, simplesmente, o fato de as EM serem atraídas para setores (ou empresas) mais produtivos (Aitken e Harrison, 1999; Sinani e Meyer, 2004). Ao permitir acompanhar o percurso das empresas nacionais ao longo do período em análise, a estimação em painel possibilita o controle dos efeitos invariantes no tempo que são específicos às empresas e que, provavelmente, estarão correlacionados com a dimensão da presença externa. Se esta questão não for controlada, os resultados obtidos poderão ser enviesados. Por outro lado, a utilização de uma desagregação setorial ou em nível de empresa/estabelecimento também pode ser relevante, na medida em que as empresas podem ser altamente heterogêneas num dado setor.

Tendo em vista realçar as possíveis divergências associadas às características empíricas dos estudos relativos à existência de externalidades intra-setoriais, os estudos foram agrupados em função do tipo de dados que utilizam (cross-section ou painel) e do nível de desagregação empregado (setorial ou por empresa). A Tabela 1 sintetiza os resultados. 
TABELA I - EXTERNALIDADES INTRA-SETORIAIS - SÍNTESE DE RESULTADOS

\begin{tabular}{|c|c|c|c|}
\hline Estudo & País & Período & Resultado \\
\hline \multicolumn{4}{|c|}{ Dados cross-section/Desagregação setorial } \\
\hline $\begin{array}{l}\text { Caves (1974) } \\
\text { Globerman (1979) } \\
\text { Blomström e Persson (1983) } \\
\text { Blomström (1986) } \\
\text { Blomström e Wolff (1994) } \\
\text { Kokko (1994) } \\
\text { Kokko (1996) } \\
\text { Imbriani e Reganati (1999) } \\
\text { Liu et al. (2001) } \\
\text { Driffield (2001) } \\
\text { Li et al. (2001) } \\
\text { Liu e Wang (2003) }\end{array}$ & $\begin{array}{l}\text { Austrália } \\
\text { Canadá } \\
\text { México } \\
\text { México } \\
\text { México } \\
\text { México } \\
\text { México } \\
\text { Itália } \\
\text { China } \\
\text { R. Unido } \\
\text { China } \\
\text { China }\end{array}$ & $\begin{array}{l}1966 \\
1972 \\
1970 \\
1970 / 75 \\
1970 / 75 \\
1970 \\
1970 \\
1992 \\
1996,1997 \\
1989 / 1992 \\
1995 \\
1995\end{array}$ & $\begin{array}{c}+ \\
+ \\
+ \\
+ \\
+ \\
+ \\
+ \\
+ \\
+ \\
\text { n.s. } \\
+ \\
+\end{array}$ \\
\hline \multicolumn{4}{|c|}{ Dados cross-section/Desagregação por empresa } \\
\hline $\begin{array}{l}\text { Kokko et al. (1996) } \\
\text { Blomström e Sjöholm (1999) } \\
\text { Chuang e Lin (1999) } \\
\text { Sjöholm (1999a) } \\
\text { Sjöholm (1999b) } \\
\text { Kokko et al. (2001) } \\
\text { Dimelis e Louri (2001) } \\
\text { Dimelis e Louri (2002) } \\
\text { Narula e Marin (2003) } \\
\text { Khawar (2003) }\end{array}$ & $\begin{array}{l}\text { Uruguai } \\
\text { Indonésia } \\
\text { Taiwan } \\
\text { Indonésia } \\
\text { Indonésia } \\
\text { Uruguai } \\
\text { Grécia } \\
\text { Grécia } \\
\text { Argentina } \\
\text { México }\end{array}$ & $\begin{array}{l}1990 \\
1991 \\
1991 \\
1980 / 1991 \\
1980 / 1991 \\
1988 \\
1997 \\
1997 \\
1992 / 1996 \\
1990\end{array}$ & $\begin{array}{c}\text { n.s. } \\
+ \\
+ \\
+ \\
+ \\
+ \\
+ \\
+ \\
\text { n.s. } \\
\text { n.s. }\end{array}$ \\
\hline \multicolumn{4}{|c|}{ Dados em painel/Desagregação setorial } \\
\hline $\begin{array}{l}\text { Santos (1991) } \\
\text { Liu (2002) } \\
\text { Kugler (2001) } \\
\text { Reis (2001) } \\
\text { Flôres et al. (2007) } \\
\text { Driffield e Love (2002) }\end{array}$ & $\begin{array}{l}\text { Portugal } \\
\text { China } \\
\text { Colômbia } \\
\text { Portugal } \\
\text { Portugal } \\
\text { R. Unido }\end{array}$ & $\begin{array}{l}1977-1982 \\
1993-1995 \\
1974-1998 \\
1995-1998 \\
1992-1995 \\
1984-1995\end{array}$ & $\begin{array}{c}+ \\
+ \\
\text { n.s. } \\
+ \\
\text { n.s. } \\
\text { n.s. }\end{array}$ \\
\hline \multicolumn{4}{|c|}{ Dados em painel/Desagregação por empresa } \\
\hline $\begin{array}{l}\text { Haddad e Harrison (1993) } \\
\text { Farinha e Mata (1996) } \\
\text { Kanturia (1998) } \\
\text { Aitken e Harrison (1999) } \\
\text { Grether (1999) } \\
\text { Kanturia (2000) } \\
\text { Ponomareva (2000) } \\
\text { Kearns (2000) } \\
\text { Girma e Wakelin (2000) } \\
\text { Djankov e Hoekman (2000) } \\
\text { Kanturia (2001) } \\
\text { Sgard (2001) } \\
\text { Blalock (2001) } \\
\text { Kinoshita (2001) } \\
\text { Braconier et al. (2001) }\end{array}$ & $\begin{array}{l}\text { Marrocos } \\
\text { Portugal } \\
\text { Índia } \\
\text { Venezuela } \\
\text { México } \\
\text { Índia } \\
\text { Rússia } \\
\text { Irlanda } \\
\text { R. Unido } \\
\text { R. Tcheca } \\
\text { İ́ndia } \\
\text { Hungria } \\
\text { Indonésia } \\
\text { R. Tcheca } \\
\text { Suécia }\end{array}$ & $\begin{array}{l}1985-1989 \\
1986-1992 \\
1984 / 85-1988 / 89 \\
1976-1989 \\
1984-1990 \\
1975 / 76-1988 / 89 \\
1993-1997 \\
1984-1998 \\
1988-1996 \\
1992-1996 \\
1975 / 76-1988 / 89 \\
1992-1999 \\
1988-1996 \\
1995-1998 \\
1976,1986,1990\end{array}$ & $\begin{array}{c}\text { n.s. } \\
\text { n.s. } \\
+ \\
- \\
- \\
- \\
+ \\
+ \\
+ \\
- \\
\text { n.s. } \\
+ \\
\text { n.s. } \\
\text { n.s. } \\
\text { n.s. }\end{array}$ \\
\hline
\end{tabular}

(continua)

Nota: n.s. - não significativo. 
TABELA 1 - EXTERNALIDADES INTRA-SETORIAIS - SÍNTESE DE RESULTADOS (cont.)

\begin{tabular}{|c|c|c|c|}
\hline Dados em painel / Desagregação por empresa & País & Período & Resultado \\
\hline \multirow[t]{3}{*}{ Konings (2001) } & Bulgária & 1993-1997 & - \\
\hline & Polônia & 1993-1997 & n.s. \\
\hline & Romênia & 1994-1997 & - \\
\hline \multirow[t]{3}{*}{ Castellani e Zanfei (2001) } & França & 1993-1997 & n.s. \\
\hline & Espanha & 1993-1997 & - \\
\hline & Itália & 1993-1997 & + \\
\hline Harris e Robinson (2002) & R. Unido & 1974-1995 & +/-/n.s. \\
\hline Kanturia (2002) & Índia & 1989/90-1996/97 & - \\
\hline Haskel et al. (2002) & R. Unido & 1973-1992 & + \\
\hline \multirow[t]{3}{*}{ Barrios et al. (2002) } & Grécia & $1992-1997$ & n.s. \\
\hline & Irlanda & 1992-1997 & n.s. \\
\hline & Espanha & 1992-1997 & n.s. \\
\hline Barrios e Strobl (2002) & Espanha & $1990-1998$ & n.s. \\
\hline Schoors e van der Tol (2002) & Hungria & $1997-1998$ & + \\
\hline Proença et al. (2002) & Portugal & 1996-1998 & n.s. \\
\hline Ruane e Ugur (2002) & Irlanda & 1991-1998 & + \\
\hline Görg e Strobl (2002) & Gana & $1991-1997$ & + \\
\hline Banga (2003) & Índia & $1993 / 94-1999 / 00$ & n.s. \\
\hline Yudaeva et al. (2003) & Rússia & $1993-1997$ & + \\
\hline Keller e Yeaple (2003) & EUA & $1987-1996$ & + \\
\hline \multirow[t]{8}{*}{ Damijan et al. (2003a) } & Bulgária & 1994-1998 & n.s. \\
\hline & R. Tcheca & 1994-1998 & - \\
\hline & Estônia & 1994-1998 & n.s. \\
\hline & Hungria & 1994-1998 & n.s. \\
\hline & Polônia & 1994-1998 & - \\
\hline & Romênia & 1994-1998 & + \\
\hline & Eslováquia & 1994-1998 & n.s. \\
\hline & Eslovênia & 1994-1998 & n.s. \\
\hline \multirow[t]{10}{*}{ Damijan et al. (2003b) } & Bulgária & 1995-1999 & n.s. \\
\hline & R. Tcheca & 1995-1999 & n.s. \\
\hline & Estônia & 1994-1998 & n.s. \\
\hline & Hungria & 1995-1999 & n.s. \\
\hline & Polônia & 1995-1999 & n.s. \\
\hline & Romênia & 1995-1999 & + \\
\hline & Eslováquia & 1995-1999 & n.s. \\
\hline & Eslovênia & 1995-1999 & n.s. \\
\hline & Letônia & 1995-1999 & n.s. \\
\hline & Lituânia & 1995-1999 & n.s. \\
\hline Sinani e Meyer (2004) & Estônia & 1994-1999 & + \\
\hline Javorcik (2004) & Lituânia & $1996-2000$ & n.s. \\
\hline \multirow[t]{5}{*}{ Torlak (2004) } & R. Tcheca & 1993-1999 & - \\
\hline & Polônia & 1993-1999 & n.s. \\
\hline & Hungria & $1994-2000$ & + \\
\hline & Romênia & 1993-1999 & - \\
\hline & Bulgária & 1993-1999 & n.s. \\
\hline Karpaty e Lundberg (2004) & Suécia & $1990-2000$ & + \\
\hline Gonçalves (2005) & Brasil & $1997-2000$ & n.s. \\
\hline Proença et al. (2006) & Portugal & $1996-1998$ & + \\
\hline
\end{tabular}

Nota: n.s. - não significativo. 


\section{(i) Dados Cross-Section / Desagregação Setorial}

As primeiras contribuições que procuraram investigar a possibilidade de a presença das EM gerar externalidades para as empresas nacionais começaram a partir de meados da década 70, com recurso a dados cross-section e desagregação setorial e incluem os estudos de Caves (1974) (com dados para a Austrália), Globerman (1979) (com informação referente ao Canadá), e Blomström e Persson (1983) e Blomström (1986) (com dados relativos ao México), todos eles identificando a existência de um efeito positivo para as empresas nacionais.

Posteriormente, outros autores, condicionados pela disponibilidade de informação estatística, seguiram esta linha de trabalho, em particular Blomström e Wolff (1994) e Kokko (1994, 1996), que se concentraram no caso mexicano. Nestes estudos, a produtividade do trabalho das empresas nacionais é positivamente afetada pela presença das EM. Kokko (1996) fornece, contudo, um importante contributo adicional, dado que é um dos poucos estudos que procura distinguir diferentes canais de geração de externalidades. Esse estudo considera que o efeito positivo potencial da presença das EM não é apenas proporcional à dimensão da sua presença no mercado nacional; é também o resultado da interação (concorrência) entre as empresas nacionais e as EM, como sugerido por Wang e Blomström (1992). Para captar este efeito, é considerado um sistema de equações simultâneas em que a produtividade das empresas nacionais é determinada não só pela dimensão da presença externa e pelos restantes fatores usualmente incluídos como variáveis de controle, como também pela produtividade das EM; por sua vez, a produtividade das EM é determinada pelos mesmos fatores que a das nacionais (com exceção, naturalmente, da presença externa). Quando é considerada uma subamostra excluindo situações de "enclave" em que não há significativa ligação entre as empresas nacionais e as EM (i.e., setores com forte presença externa e muito concentrados), verifica-se que a eficiência das empresas nacionais é determinada pela presença externa e pelo efeito de concorrência. ${ }^{8}$

Li et al. (2001) adotam, para o caso chinês, uma abordagem semelhante a Kokko (1996), embora mais detalhada, pois são considerados três grupos de empresas: empresas multinacionais, empresas estatais e "outras empresas locais". O impacto da presença externa é significativo e positivo no caso das empresas locais não estatais, sendo não significativo o efeito sobre a eficiência das empresas estatais. Quando se avalia o efeito de concorrência das EM, verifica-se que este é diferenciado consoante o grupo de empresas, sendo positivo nas empresas estatais e negativo nas restantes empresas nacionais.

8 A relevância do canal de concorrência é igualmente destacada por Driffield (2001), tendo por suporte informação estatística para o caso do Reino Unido. 
A evidência favorável à ocorrência de externalidades do IDE para as empresas nacionais é ainda extensível aos trabalhos de Imbriani e Reganati (1999), baseado no caso italiano, e de Liu e Wang (2003), para a economia chinesa. ${ }^{9}$

Em suma, os resultados obtidos com este tipo de dados sugerem, com clareza, a existência de externalidades positivas do IDE para as empresas nacionais.

\section{(ii) Dados Cross-Section / Desagregação por Empresa}

O desenvolvimento de novas bases de dados - mais detalhadas - permitiu a concretização de um largo conjunto de trabalhos que, embora mantendo uma análise cross-section, utilizam informação desagregada no plano da empresa/estabelecimento. Consideremos, brevemente, as conclusões passíveis de serem extraídas deste leque de estudos.

Sjöholm (1999a,b) debruça-se sobre o caso da Indonésia, tendo por suporte informação estatística que abrange 329 setores com um total de 8.086 empresas em 1980 e 16.382 empresas em 1991. Ambos os estudos avaliam o impacto tanto no nível de produtividade do trabalho como no seu crescimento, concluindo favoravelmente à existência de externalidades (intra-setoriais) do IDE. ${ }^{10} \mathrm{~A}$ mesma conclusão decorre do estudo de Blomström e Sjöholm (1999) relativo ao caso indonésio.

Kokko et al. (1996, 2001) analisam o caso da economia uruguaia. No primeiro estudo, considerando dados relativos a 159 estabelecimentos, não é detectada influência significativa da presença externa sobre a produtividade do trabalho dos estabelecimentos nacionais. No entanto, o segundo trabalho toma por suporte informação relativa a 763 empresas domésticas pertencentes a setores onde existe presença externa e indica a existência de externalidades positivas do IDE. O mesmo impacto favorável é detectável nos trabalhos de Chuang e Lin (1999) e Dimelis e Louri (2002), referentes, respectivamente, a Taiwan e à Grécia.

No entanto, resultados distintos são também identificáveis na literatura, como se observa em Khawar (2003), numa análise relativa ao México, e em Narula e Marin (2003), no caso argentino. Ambos concluem que a influência da presença externa na eficiência das empresas nacionais não é significativa.

Os resultados decorrentes deste grupo de trabalhos não oferecem, portanto, suporte inequívoco ao efeito positivo esperado. Enquanto em alguns deles se identificam externalidades positivas, outros não confirmam essa hipótese, obtendo resultados não significativos.

9 Este último estudo circunscreve-se ao caso do setor eletrônico.

10 No caso de Sjöholm (1999b), estamos considerando a regressão efetuada em nível nacional. 


\section{(iii) Dados em Painel / Desagregação Setorial}

A consideração de dados em painel com uma desagregação setorial é realizada por um grupo relativamente limitado de estudos. Entre estes, inclui-se a investigação desenvolvida por Liu (2002), com referência à economia chinesa. Os resultados obtidos indicam a ocorrência de externalidades positivas. A mesma conclusão emerge dos trabalhos, baseados no caso português, de Reis (2001) e Santos (1991), embora neste último caso a evidência se revele fraca. ${ }^{11}$

No entanto, um impacto não significativo é detectado nos estudos desenvolvidos por Flôres et al. (2007) para Portugal, Driffield e Love (2002) para o Reino Unido, e Kugler (2001) para a Colômbia.

As conclusões emergentes deste grupo de estudos - um impacto positivo ou uma influência não significativa - parecem, portanto, replicar as obtidas no contexto do grupo precedente.

\section{(iv) Dados em Painel / Desagregação por Empresa}

Os dois estudos pioneiros com recurso a dados em painel e desagregação por empresa vieram a ter uma importância decisiva para o prosseguimento da investigação nesta área. Referimo-nos aos trabalhos de Haddad e Harrison (1993), relativos à economia marroquina, e ao de Aitken e Harrison (1999) para a Venezuela. No primeiro, embora nos setores com presença externa mais significativa exista menor dispersão dos níveis de produtividade, não se identifica nenhuma influência significativa dessa mesma presença sobre o crescimento da produtividade das empresas nacionais. Quanto ao segundo, detecta-se uma influência negativa da presença externa sobre a produtividade nacional, explicável, segundo os autores, pelo efeito negativo da concorrência.

A não confirmação da existência de externalidades positivas do IDE nestes dois estudos conduziu à generalização da hipótese de os resultados anteriores derivarem mais do tipo de dados usados do que de uma realidade concreta, principalmente levando em consideração as indiscutíveis vantagens da utilização de dados em painel desagregados por empresa. Em face disto, não surpreende a emergência de um vasto leque de estudos que, para diferentes espaços econômicos e lapsos temporais distintos, procuraram reproduzir a análise anterior com este tipo de dados.

Alguns dos trabalhos empreendidos neste novo grupo de estudos abarcam evidência relativa a mais de um país, o que tem a vantagem de limitar (ou, em certos casos, eli-

11 A conclusão do autor aponta para a inexistência de um efeito significativo. No entanto, esta conclusão funda-se numa análise baseada num limiar de $5 \%$ para os coeficientes serem significativos. Pelas razões explicitadas anteriormente, fazemos a interpretação com base num critério de $10 \%$. 
minar) as divergências atribuíveis à diferença de especificação, permitindo um nível superior de comparabilidade entre os resultados. Neste subgrupo de estudos, encontramse os trabalhos de Konings (2001), Castellani e Zanfei (2001), Barrios et al. (2002), Damijan et al. (2003a,b) e Torlak (2004). No primeiro, são considerados três países - Bulgária, Polônia e Romênia -, sendo detectadas externalidades negativas, no plano setorial, nos casos búlgaro e romeno, enquanto a evidência para a economia polonesa não produz, a este respeito, resultados significativos. O estudo de Castellani e Zanfei (2001) analisa a existência de externalidades do IDE em três países - França, Espanha e Itália -, caracterizando-se os resultados obtidos pela heterogeneidade, uma vez que é encontrado um impacto positivo no caso da economia italiana, negativo na economia espanhola e não significativo no caso francês. Barrios et al. (2002) abordam evidência referente a Espanha, Grécia e Irlanda, criando uma base de dados comparável para os três países, e concluem pela inexistência de externalidades em quaisquer dos países considerados, o que corrobora o resultado obtido por Barrios e Strobl (2002) para a economia espanhola. Damijan et al. $(2003 \mathrm{a}, \mathrm{b})$ concentram a sua avaliação num largo conjunto de Países da Europa Central e Oriental (PECO). No primeiro estudo destes autores, são considerados oito países - Bulgária, República Tcheca, Estônia, Hungria, Polônia, Romênia, Eslováquia e Eslovênia -, com a inclusão neste grupo de Letônia e Lituânia no segundo estudo. No seu conjunto, os resultados são robustos, pois apenas no caso romeno são detectadas externalidades positivas; nas situações restantes, a influência é considerada não significativa, exceção feita aos casos da República Tcheca e da Polônia, onde, surpreendentemente - dado o seu maior nível de desenvolvimento - é encontrado um impacto negativo. ${ }^{12}$ Torlak (2004) obtém evidência relativa a cinco países - República Tcheca, Polônia, Hungria, Romênia e Bulgária - e os resultados também não suportam a hipótese de externalidades positivas do IDE para as empresas nacionais: em dois dos países considerados - Polônia e Bulgária - o impacto não é estatisticamente significativo, em outros dois - República Tcheca e Romênia - a influência é mesmo negativa e significativa e apenas na Hungria é encontrada evidência favorável à hipótese de ocorrência do fenômeno.

Apesar de não serem absolutamente concordantes, os estudos mencionados parecem confirmar, portanto, as dúvidas levantadas pelas investigações pioneiras de Haddad e Harisson (1993) e Aitken e Harisson (1999).

Essa mesma realidade emerge de estudos que abordam evidência referente apenas a um país. Por exemplo, nos trabalhos de Djankov e Hoekman (2000) para a República Tcheca, e de Grether (1999) para o México, são detectadas externalidades negativas do IDE, enquanto em muitos outros a influência é considerada não significativa, como acontece em Blalock (2001) para a Indonésia, Kinoshita (2001) para a República

12 Neste caso, consideramos a regressão que controla a capacidade de absorção. 
Tcheca, Banga (2003) para a Índia, Javorcik (2004) para a Lituânia, Proença et al. (2002) para Portugal e Gonçalves (2005) para o Brasil. ${ }^{13}$

Perante esta evidência, tem emergido na literatura sobre esta questão uma visão reticente quanto à existência de externalidades do IDE expressa, por exemplo, nos surveys de Görg e Strobl (2001) e Görg e Greenaway (2004).

Note-se, contudo, que é também possível encontrar um outro leque de estudos com este tipo de dados que identifica um impacto positivo da presença externa, constituindo exemplo os trabalhos de Haskel et al. (2002) para o Reino Unido, Keller e Yeaple (2003) para os EUA, Ponomareva (2000) e Yudaeva et al. (2003) para a Rússia, Kearns (2000) e Ruane e Ugur (2002) ${ }^{14}$ para a Irlanda, Sgard (2001) e Schoors e van der Tol (2002) para a Hungria, Karpaty e Lundberg (2004) para a Suécia, Sinani e Meyer (2004) para a Estônia e Proença et al. (2006) para Portugal. ${ }^{15}$

A heterogeneidade que se observa no vasto conjunto de estudos já mencionados é ainda patente na sequiência de análises desenvolvidas para a Índia por Kanturia Kanturia (1998, 2000, 2001, 2002) -, através do recurso a diferentes especificações e espaços temporais alternativos, assim como nas conclusões de Harris e Robinson (2002) para o Reino Unido. Este último estudo tem a particularidade de proceder à análise da existência de externalidades desagregando setorialmente (20 setores); os resultados obtidos registram efeitos positivos, negativos e não significativos conforme os setores considerados, mas não se encontra uma relação direta com características próprias dos setores.

Consideremos, finalmente, o trabalho de Görg e Strobl (2002), cuja especificidade reside na tentativa de isolar o efeito de externalidade suscitado pela mobilidade dos trabalhadores. Baseando a análise na economia do Gana, procuram investigar se empresas nacionais cujos empresários tiveram experiência em empresas multinacionais possuem vantagem em termos de produtividade face às restantes. Os resultados mostram que, efetivamente, as empresas constituídas por empresários que adquiriram experiência trabalhando em empresas multinacionais evidenciam um crescimento superior da sua produtividade, mas tal efeito circunscreve-se à situação em que a experiência na EM ocorreu no mesmo setor de atividade. Quando ocorre em outro setor, o efeito é não significativo (ou, em algumas especificações, mesmo negativo),

13 Num outro estudo tomando por base a economia brasileira, Araújo (2005) encontra evidência mais favorável à ocorrência de efeitos positivos da presença externa para as empresas nacionais. A metodologia não é, contudo, plenamente comparável com a dos estudos que servem de suporte à análise desenvolvida no presente trabalho, razão pela qual o excluímos da Tabela 1.

14 Consideramos, neste caso, as regressões que assumem a presença externa calculada segundo a proposta de Castellani e Zanfei (2002).

15 Este último estudo oferece, ainda, um contributo adicional ao sugerir que as técnicas tradicionais de estimação em painel poderão conduzir a uma séria subavaliação dos reais efeitos de externalidade do IDE para as empresas nacionais. 
exprimindo a dificuldade de os empresários utilizarem os conhecimentos adquiridos num setor diferente.

\subsection{Externalidades Inter-Setoriais do IDE}

A generalidade dos estudos que tem investigado a existência de externalidades do IDE limita a sua avaliação às externalidades intra-setoriais. Só recentemente emergiu uma vertente de análise visando averiguar a ocorrência de externalidades inter-setoriais. A razão central da escassa atenção dedicada a esta vertente resulta da dificuldade associada à quantificação dos efeitos inter-setoriais. $\mathrm{O}$ surgimento de bases de dados mais detalhadas facilitou, porém, o desenvolvimento deste tipo de estudos.

Alguns autores têm argumentado que a existência de externalidades inter-setoriais é, à luz das previsões teóricas, mais justificável do que as externalidades intra-setoriais. $\mathrm{O}$ argumento central funda-se, por um lado, no fato de o principal (embora não único) fator negativo identificado na literatura estar associado ao efeito de concorrência e este assumir uma característica essencialmente intra-setorial. Por outro lado, é nas relações com fornecedores e compradores locais que as EM têm maior estímulo em cooperar, facilitando, assim, ganhos de eficiência para as empresas nacionais. ${ }^{16}$ A Tabela 2 sintetiza a evidência existente em relação às externalidades inter-setoriais. ${ }^{17}$

16 Ver Kugler (2001), para uma discussão detalhada desta argumentação.

17 Não incluímos na Tabela 2 o estudo de Sjöholm (1999b) para a Indonésia, dado que a proxy para o efeito inter-setorial avalia essencialmente o efeito de aglomeração, na medida em que mede a proporção da presença estrangeira na globalidade dos setores restantes e na região. Desta forma, não difere significativamente da que é usada por Harris e Robinson (2002), Haskel et al. (2002) ou Konings (2001) para captar o efeito de aglomeração. Não consideramos, igualmente, o estudo de Harris e Robinson (2002), pelo fato de a medida da presença externa em nível inter-setorial não ser obtida como uma média ponderada da presença nos setores restantes, mas antes individualizada para cada um deles; existe portanto, não apenas uma variável que capta os efeitos inter-setoriais mas tantas quanto o número de indústrias consideradas (excluindo aquela a que pertence a empresa). 
TABELA 2 - EXTERNALIDADES INTER-SETORIAIS - SÍNTESE DE RESULTADOS

\begin{tabular}{|c|c|c|c|c|c|}
\hline \multirow[t]{2}{*}{ Estudo } & \multirow[t]{2}{*}{ País } & \multirow[t]{2}{*}{ Período } & \multicolumn{3}{|c|}{ Resultado } \\
\hline & & & Global & Backward & Forward \\
\hline Blalock (2001) & Indonésia & 1988-1996 & & + & \\
\hline Kugler (2001) & Colômbia & 1974-1998 & n.s. & +(vários & - \\
\hline Barrios e Strobl (2002) & Espanha & 1990-1998 & & casos) & - \\
\hline Yudaeva et al. (2003) & Rússia & 1993-1997 & & - & - \\
\hline Schoors e van der Tol (2002) & Hungria & $1997-1998$ & & + & \\
\hline \multirow[t]{10}{*}{ Damijan et al. (2003b) } & Bulgária & 1995-1999 & & n.s. & \\
\hline & R. Tcheca & 1995-1999 & & + & \\
\hline & Estônia & 1994-1998 & & n.s. & \\
\hline & Hungria & 1995-1999 & & n.s. & \\
\hline & Polônia & 1995-1999 & & + & \\
\hline & Romênia & 1995-1999 & & n.s. & \\
\hline & Eslováquia & 1995-1999 & & + & \\
\hline & Eslovênia & 1994-1999 & & n.s. & \\
\hline & Letônia & 1994-1999 & & n.s. & \\
\hline & Lituânia & 1994-1999 & & n.s. & \\
\hline Javorcik (2004) & Lituânia & $1996-2000$ & & + & \\
\hline Gonçalves (2005) & Brasil & $1997-2000$ & & + & \\
\hline
\end{tabular}

Nota: n.s. - não significativo

À semelhança do registrado no caso das externalidades intra-setoriais, os resultados decorrentes desta vertente de avaliação não são plenamente conclusivos. Assim, a existência de externalidades inter-setoriais positivas através de backward linkages só é confirmada em metade dos casos referidos na Tabela 2. Já o impacto através de forward linkages é homogêneo em todos os estudos que o consideram. Contudo, é necessário destacar o fato de este último efeito ser negativo, em oposição ao esperado, assinalando que a produtividade das empresas nacionais é prejudicada com a aquisição de insumos às EM.

\section{QUE PERSPECTIVAS PARA A INVESTIGAÇÃO SOBRE EXTERNALIDADES DO IDE?}

Da observação dos resultados sumariados não decorre uma visão tão pessimista sobre o impacto das EM na eficiência das empresas nacionais como a de alguns surveys recentes (Görg e Strobl, 2001; Görg e Greenaway, 2004), que tomam como referência um número inferior de estudos. Contudo, a evidência exposta nas seções precedentes também não fornece um suporte claro quanto à ocorrência de externalidades do IDE. 
Tomando por referência os estudos desenvolvidos sobre externalidades intra-setoriais e, entre estes, apenas os que recorrem a dados em painel e com desagregação no plano da empresa/estabelecimento, na medida em que utilizam um tipo de dados mais apropriado, é possível constatar que, em 59 situações analisadas, ${ }^{18} 31$ encontram um impacto não significativo, 16 um impacto positivo e, em 12 casos, é detectado um efeito negativo. Em termos dos resultados empíricos, esta ambigüidade é extensiva aos estudos sobre externalidades inter-setoriais.

Enquanto os resultados sumariados neste e nos outros surveys acima mencionados resultam de uma avaliação do efeito para o conjunto das empresas da amostra estudada, é plausível que a ocorrência de externalidades do IDE para as empresas nacionais se verifique apenas para um subgrupo de empresas nacionais, aquelas que verificam determinadas condições.

De fato, na literatura recente sobre este tema é possível encontrar diversos testes a fatores condicionantes da ocorrência de externalidades, incluindo vários dos estudos que constam das Tabelas 1 e $2 .{ }^{19}$ Em geral, estes fatores são analisados de forma isolada ou, quando muito, em escasso número, não existindo, por enquanto, uma preocupação de testar de forma sistemática e integrada o conjunto de fatores já evidenciados como potencialmente relevantes. Apesar disso, os resultados obtidos são suficientemente esclarecedores da importância desta via de investigação.

Dois fatores determinantes das externalidades do IDE têm merecido especial atenção. Por um lado, argumenta-se que a difusão tecnológica não se processa de forma automática; é necessário, pelo contrário, que a empresa receptora possua a capacidade de "absorver" a nova tecnologia. Neste nível tem sido verificada a importância fulcral da dimensão do gap tecnológico entre as EM e as empresas nacionais: se for demasiado pequeno, significará que os dois tipos de empresas apresentam níveis aproximados de eficiência e, nesse caso, as empresas nacionais terão provavelmente pouco a aprender com as EM; se for demasiado amplo, as empresas nacionais não terão a "capacidade de absorção" necessária para incorporar os benefícios gerados pela presença das empresas estrangeiras. Por outro lado, tem sido defendida a existência de uma dimensão regional/local na ocorrência de externalidades, de tal forma que seriam as empresas nacionais geograficamente mais próximas das EM as que se beneficiariam de modo mais significativo. A análise empírica desenvolvida em relação a estes dois fatores confere um sólido suporte ao primeiro argumento (Kinoshita, 2001; Schoors e van der Tol, 2002; Kanturia, 2002; Keller e Yeaple, 2003; Girma, 2003; Karpaty e Lundberg, 2004), mas é mais ambígua no que se refere ao segundo fator (Sjöholm,

18 Excluindo o trabalho de Harris e Robinson (2002), dado que, por suas características particulares, não permite obter um sinal para o conjunto da amostra.

19 Para um survey sobre este tópico, veja-se Crespo e Fontoura (2007). 
1999b; Aitken e Harrison,1999; Girma e Wakelin, 2000; Girma, 2003; Yudaeva et al., 2003; Torlak, 2004).

Quanto aos fatores restantes que podemos identificar na literatura teórica e empírica sobre este tema como possíveis condicionantes das externalidades do IDE, a avaliação empírica é, por enquanto, escassa ou mesmo nula. É o caso dos seguintes: a dimensão das empresas nacionais receptoras (Aitken e Harrison, 1999; Sinani e Meyer, 2004), o fato de as empresas nacionais serem ou não exportadoras (Blomström e Sjöholm, 1999; Ponomareva, 2000; Schoors e van der Tol, 2002), o país de origem do IDE (Haskel et al., 2002; Banga, 2003; Karpaty e Lundberg, 2004), o grau de participação externa nos projetos de investimento (Blomström e Sjöholm, 1999; Dimelis e Louri, 2002; Javorcik, 2004), o modo de entrada do IDE (Braconier et al., 2001), a política comercial adotada pelo país receptor (Kokko et al., 2001), a existência de direitos de propriedade intelectual, a dimensão da utilização de insumos intermédios por parte das empresas nacionais, o tipo de formação fornecido pelas EM aos seus trabalhadores (Fosfuri et al., 2001), as formas assumidas pelos contratos entre as empresas nacionais e as EM, o nível de concorrência (Wang; Blomström, 1992), o "valor" da tecnologia introduzida pelas EM (Blomström et al., 2000) ou a motivação do IDE (Fosfuri e Motta, 1999; Driffield e Love, 2002).

Apesar das limitações atuais da pesquisa sobre os fatores determinantes destas externalidades do IDE, ela nos dá fortes razões para suspeitar que os resultados acima apresentados com base no efeito global - i.e., o efeito no conjunto das empresas consideradas na amostra estudada - estarão enviesados em relação à realidade. Não podemos, por isso, excluir a possibilidade de esses resultados "globais" estarem subavaliados nos casos em que não foram detectadas externalidades positivas significativas. ${ }^{20}$

Parece claro, portanto, que a principal via para o desenvolvimento da investigação relativa à existência de externalidades do IDE para as empresas nacionais deverá incidir na avaliação detalhada - no plano teórico e empírico - dos fatores que condicionam a ocorrência, a magnitude e o sinal das externalidades do IDE. Esta mesma mensagem emerge das palavras de Lipsey quando refere que "o que é mais necessário é uma acrescida atenção às várias circunstâncias e politicas dos paises, setores e empresas que promovem ou obstruem a existência de externalidades” (Lipsey, 2002, p. 32).

No contexto desta segunda via de análise, cremos ser ainda importante aprofundar a investigação em torno de quatro vertentes. Em primeiro lugar, é necessária uma atenção teórica maior aos canais geradores de externalidades, em particular no que se refere à análise das externalidades inter-setoriais. Em segundo lugar, é fundamental a

20 Proença et al (2006) sugerem ainda a possibilidade de os métodos econométricos usados nos estudos anteriores não serem os mais adequados. Os resultados obtidos podem, também por esta via, estar enviesados em relação à realidade. 
realização de um esforço de harmonização dos pressupostos subjacentes aos diversos estudos empíricos de forma a aumentar o seu grau de compatibilidade. Um estudo neste segundo domínio já foi iniciado através da construção de bases de dados harmonizadas para vários países. Um exemplo desse esforço é o trabalho desenvolvido por Barrios et al. (2002). Em terceiro lugar, como decorre da apresentação acima realizada, é óbvia a necessidade de maior enfoque na avaliação empírica da existência de externalidades inter-setoriais. Em quarto lugar, a realização de estudos de caso sobre a forma como as EM e as empresas nacionais se relacionam e como se podem gerar ganhos de eficiência para estas últimas poderá contribuir significativamente para o conhecimento dos mecanismos/condicionantes de transferência tecnológica entre os dois grupos de empresas.

\section{OBSERVAÇÕES FINAIS}

Diversas mensagens importantes podem ser retidas da análise efetuada neste artigo.

Salientaríamos, em primeiro lugar, o fato de, contrariamente à visão mais difundida, o impacto teórico esperado da presença externa sobre a produtividade das empresas nacionais ser ambíguo, dada a existência de múltiplos efeitos de sentido oposto e o desconhecimento das forças prevalecentes em cada situação particular.

Apesar do elevado número de estudos empíricos sobre estes efeitos do IDE nos últimos 30 anos, este survey evidencia que os resultados existentes também não fornecem indicações claras sobre o fenômeno. Enquanto a evidência pioneira sobre esta temática sugeria a existência de efeitos positivos das externalidades das EM para a produtividade das empresas nacionais no seu conjunto, estudos mais recentes, utilizando técnicas econométricas mais adequadas, têm questionado o consenso desta conclusão. De fato, os resultados sumariados estão longe de confirmar inequivocamente tais efeitos - conforme atesta a obtenção de resultados de sinal oposto segundo os estudos - de muitos resultados não significativos e, também, a ocorrência de diversos sinais negativos.

Para um entendimento mais cabal da relação entre as EM e a eficiência das empresas nacionais, sugere-se que a avaliação empírica incida sobretudo no estudo detalhado da influência dos fatores condicionantes da existência, da dimensão e do sinal das externalidades do IDE.

O reconhecimento da necessidade de aprofundamento desta segunda via de investigação dos efeitos indiretos do IDE tem implicaçóes importantes para uma adequada definição das políticas de atração do IDE: sem um estudo detalhado dos fatores condicionantes destes efeitos e conseqüente identificação das características do IDE 
que convém ao país receptor, as eventuais políticas que fomentem as entradas de IDE poderão produzir efeitos na produtividade das empresas nacionais não só irrelevantes como perversos, caso originem a sua redução.

Seja a motivação para a investigação destas externalidades do IDE uma definição mais adequada de políticas econômicas relacionadas com as entradas de IDE ou, simplesmente, um melhor conhecimento da realidade, um balanço geral decorrente do acima exposto é que, com três décadas voltadas ao estudo pioneiro de Caves (1974), há ainda um vasto esforço a ser desenvolvido na busca de respostas às questões que se levantam sobre a importância da presença das EM para a eficiência das empresas nacionais.

\section{REFERENNCIAS BIBLIOGRÁFICAS}

Aitken, B.; G. Hanson, G.; Harrison, A. Spillovers, foreign investment and export behaviour. Journal of International Economics, v. 43, p. 103-132, 1997.

; Harrison, A. Do domestic firms benefit from direct foreign investment? Evidence from Venezuela. American Economic Review, v. 89, n.3, p. 605-618, 1999.

Araújo, R. Esforços tecnológicos das empresas transnacionais e domésticas. In: J. De Negri, J.; Salerno, M. (eds.), Inovaçôes, padrôes tecnológicos e desempenho das firmas industriais brasileiras. Brasília: IPEA, 2005.

Banga, R. Do productivity spillovers from japanese and U.S. FDI differ?.Delhi School of Economics, 2003. Mimeografado.

Barrios, S.; Dimelis, S.; Louri, H.; Strobl, E. Efficiency spillovers from foreign direct investment in the EU periphery: a comparative study of Greece, Ireland and Spain. FEDEA Working Paper 2002-02, 2002.

; Strobl, E. Foreign direct investment and productivity spillovers: evidence from the spanish experience. Weltwirtschaftliches Archiv, v.138, n.3, p.459- 481, 2002.

Bernard, A.; Jensen, J. Exceptional exporters' performance: cause, effect or both. Journal of International Economics, 47, p. 1-25, 1999.

Blalock, G. Technology from foreign direct investment: strategic transfer through supply chains. Artigo apresentado na Conferência Empirical Investigations in International Trade Conference, Purdue University, nov. 2001.

Blomström, M. Foreign investment and productive efficiency: the case of Mexico. Journal of Industrial Economics, v.35, n.1, p. 97-110, 1986.

; Kokko, A. Human capital and inward FDI. European Institute of Japanese Studies. Working Paper n. 167, 2003 
; Globerman, S.; Kokko, A. The determinants of host country spillovers from foreign direct investment. CEPR Discussion Paper 2350, 2000.

; Kokko, A. Multinational corporations and spillovers. Journal of Economic Surveys, v.12, n.3, p. 247-277, 1998.

; Persson, H. Foreign investment and spillover efficiency in an underdeveloped economy: evidence from the mexican manufacturing industry. World Development, v.11, n.6, p. 493-501, 1983.

; Sjöholm, F. Technology Transfer and Spillovers: Does Local Participation With Multinationals Matter? European Economic Review, 43, p. 915-923, 1999.

; Wolff, E. Multinationals corporations and productive convergence in Mexico. In: Baumol, W.; Nelson, R.; Wolff, E. (eds.), Convergence of productivity: cross national studies and historical evidence. Oxford: Oxford University Press, p. $263-283,1994$.

Braconier, H.; Ekholm, K.; Midelfart-Knarvik, K. In search of FDI - transmitted R\&D spillovers: a study based on swedish data. Weltwirtschaftliches Archiv, v.137, n. 4, p. 644-665, 2001.

Castellani, D.; Zanfei, A. Productivity gaps, inward investments and productivity of european firms. University of Urbino, 2001. Mimeografado.

Multinationals companies and productivity spillovers: is there a specification error?. University of Urbino, 2002. Mimeografado.

Caves, R. Multinational firms, competition and productivity in host-country markets. Economica, 41, p. 176-193, 1974.

. Multinational enterprise and economic analysis. New York: Cambridge University Press, 1996.

Chuang, Y.; Lin, C. Foreign direct investment, R\&D and spillover efficiency: evidence from Taiwan's manufacturing firms. Journal of Development Studies, v.35, n.4, p. 117- 137, 1999.

Crespo, N.; Fontoura, M. Determinant Factors of FDI Spillovers - What Do We Really Know? World Development, v. 35, n.3, p.410-425, 2007.

Damijan, J.; Knell, M.; Majcen, B.; Rojec. M. The role of FDI, R\&D accumulation and trade in transferring technology to transition countries: evidence from firm level panel data for eight transition countries. Economic Systems, 27, p. 189 $-204,2003 a$.

Technology transfer through FDI in top-10 transition countries: how important are direct effects, horizontal and vertical spillovers? 2003b. (William Davidson Working Paper 549).

Das, S. Externalities and technology transfer through multinationals corporations - a theoretical analysis. Journal of International Economics, 22, p. 171-182, 1987. 
Dimelis, S.; Louri, H. Foreign direct investment and efficiency benefits: do size and ownership matter? Artigo apresentado na Conferência Business \& Economics Society International Conference. Paris, jul. 2001.

. Foreign ownership and production efficiency: a quantile regression analysis. Oxford Economic Papers, 54, p. 449 - 469, 2002.

Djankov, S.; Hoekman, B. Foreign investment and productivity growth in czech enterprises”. The World Bank Economic Review, 14(1), p. 49-64, 2000.

Driffield, $\mathrm{N}$. The impact on domestic productivity of inward investment in the UK. The Manchester School, 69 (1), p. 103 -119, 2001.

.; Love, J. Does the motivation for foreign direct investment affect productivity spillovers to the domestic sector?.University of Birmingham, 2002. Mimeografado.

Farinha, L.; Mata, J. The impact of foreign direct investment in the portuguese economy. Banco de Portugal Working Paper $n^{0}$ 16/96, 1996.

Flôres, R.; Fontoura, M.; Santos, R. Foreign direct investment and spillovers: additional lessons from a country study. The European Journal of Development Research, v. 19, n. 3, p. 372-390, 2007.

Fosfuri, A.; Motta, M. Multinationals without advantages. Scandinavian Journal of Economics, 101(4), p. 617-630, 1999.

; Ronde; T. Foreign direct investment and spillovers through workers' mobility. Journal of International Economics, 53, p. 205-222, 2001.

Gershenberg, I. The training and spread of managerial know-how - a comparative analysis of multinationals and other firms in Kenya. World Development, 15 (7), p. 931-939, 1987.

Girma, S. Absorptive capacity and productivity spillovers from FDI: a threshold regression analysis. European Economy Group, 2003 (Working Paper No. 25/2003).

; Wakelin, K. Are there regional spillovers from FDI in the UK?. Artigo apresentado na International Economics Association Conference. Nottingham, julho de 2000.

Glass, A.; Saggi, K. Multinational firms and technology transfer. Scandinavian Journal of Economics, 104 (4), p. 495- 513, 2002.

Globerman, S. Foreign direct investment and spillover efficiency benefits in canadian manufacturing industries. Canadian Journal of Economics, 12(1), p. 42-56, 1979.

Gonçalves, J. Empresas estrangeiras e transbordamentos de produtividade na induistria brasileira: 1997 - 2000. Rio de Janeiro: BNDES, 2005.

Gonçalves, R. Technological spillovers and manpower training: a comparative analysis of multinational and national enterprises in brazilian manufacturing. Journal of Development Economics, 11, p. 119-132, 1986. 
Görg, H.; Greenaway, D. Much ado about nothing? do domestic firms really benefit from foreign direct investment?. World Bank Research Observer, 19 (2), p. 171197, 2004.

; Strobl, E. Multinational companies and productivity spillovers: a metaanalysis. Economic Joumal, 111, p. 723-739, 2001.

; Spillovers from foreign firms through worker mobility: an empirical investigation. University of Nottingham, 2002. Mimeografado.

Greenaway, D.; Sousa, N.; Wakelin, K. Do domestic firms learn to export from multinationals? European Journal of Political Economy, 20, p. 1027-1043, 2004.

Grether, J. Determinants of technological diffusion in mexican manufacturing: a plant-level analysis. World Development, 27(7), p. 1287 - 1298, 1999.

Haddad, M.; Harrison, A. Are there positive spillovers from direct foreign investment? Evidence from panel data for Morocco. Journal of Development Economics, 42 , p. $51-74,1993$.

Hanson, G. Should countries promote foreign direct investment? Nações Unidas, 2001. (G-24 Discussion Paper No. 9)

Harris, R.; Robinson, C. Spillovers from foreign ownership in the United Kingdom - estimates for UK manufacturing using the ARD. Artigo apresentado na RES 2002 Conference, 2002.

Harrison, A. Productivity, imperfect competition and trade reform. Journal of International Economics, 36, p. 53-73, 1994.

Haskel, J.; Pereira, S.; Slaughter, M. Does inward foreign direct investment boost the productivity of domestic firms? Department of Economics, Queen Mary, University of London, 2002. (Working Paper No. 452).

Imbriani, C.; Reganati, F. Productivity spillovers and regional differences: some evidence on the italian manufacturing sector. Centro di Economia del Lavoro e di Politica Economica, Università degli Studi di Salerno, 1999 (Discussion Paper 48)

Javorcik, B. Does foreign direct investment increase the productivity of domestic firms? In search of spillovers through backward linkages. American Economic Review, 94(3), p. 605-627, 2004.

Kanturia, V. Technology transfer and spillovers for indian manufacturing firms $D e$ velopment Policy Review, 16(1), p. 73-91, 1998.

; Productivity spillovers from technology transfer to indian manufacturing firms. Journal of International Development, 12, p. 343-369, 2000.

; Foreign firms, technology transfer and knowledge spillovers to indian manufacturing firms: a stochastic frontier analysis. Applied Economics, 33, p. 625-642, 2001.

; Liberalisation, FDI, and productivity spillovers - an analysis of indian manufacturing firms. Oxford Economic Papers, 54, p. 688-718, 2002. 
Karpaty, P.; Lundberg, L. Foreign direct investment and productivity spillovers in swedish manufacturing. FIEF Working Paper Series, No. 194, 2004.

Kearns, A. Essays on the consequences of research and development for manufacturing firms in Ireland. 2000. Tese (Doutoramento), Department of Economics, University of Dublin, Trinity College.

Keller, W.; Yeaple, S. Multinational enterprises, international trade, and productivity growth: firm-level evidence from the United States. NBER Working Paper No. 9504, 2003.

Khawar, M. Productivity and foreign direct investment - evidence from Mexico. Journal of Economic Studies, 30(1), p. 66-76, 2003.

Kinoshita, Y. R\&D and technology spillovers through FDI: innovation and absorptive capacity. CEPR Discussion Paper No. 2775, 2001.

Kokko, A. Technology, market characteristics, and spillovers. Journal of Development Economics, 43, p. 279-293, 1994.

; Productivity spillovers from competition between local firms and foreign affiliates. Journal of International Development, 8(4) , p. 517-530, 1996.

; Blomström, M. Policies to encourage inflows of technology through foreign multinationals. World Development, 23(3), p. 459-468, 1995.

; Tansini, R.; Zejan, M. Local technological capability and productivity spillovers from FDI in the uruguayan manufacturing sector. Journal of Development Studies, 32(4) , p. $602-611,1996$.

; Trade regimes and spillover effects of FDI: evidence from Uruguay. Weltwirtschaftliches Archiv, 137(1), p. 124-149, 2001.

Konings, J. The effects of foreign direct investment on domestic firms: evidence from firm-level panel data in emerging economies. Economics of Transition, 9(3), p. 619- 633, 2001.

Kugler, M. The diffusion of externalities from foreign direct investment: theory ahead of measurement. University of Southampton, 2001. Mimeografado.

Lall, S. Vertical interfirm linkages in LDCs: an empirical study. Oxford Bulletin of Economics and Statistics, 42 (3), p. 203-226, 1980.

Li, X.; Liu, X.; Parker, D. Foreign direct investment and productivity spillovers in the chinese manufacturing sector. Economic Systems, 25, p. 305-321, 2001.

Lin, P.; Saggi, K. Multinational firms and backward linkages: a survey and a simple model. Lingnan University and Southern Methodist University, 2004. Mimeografado.

Lipsey, R. Home and host country effects of FDI. NBER Working Paper 9293, 2002. 
Liu, X.; Parker, D.; Vaidya, K.; Wei, Y. The impact of foreign direct investment on labour productivity in the chinese electronics industry. International Business Review, 10, p. 421- 439, 2001.

; Wang, C. Does foreign direct investment facilitate technological progress? Evidence from chinese industries. Research Policy, 32, p. 945-953, 2003.

Liu, Z. Foreign direct investment and technology spillover: evidence from China. Journal of Comparative Economics, 30(3), p. 579-602, 2002.

Markusen, J.; Venables, A. Foreign direct investment as a catalyst for industrial development. European Economic Review, 43, p. 335-356, 1999.

Narula, R.; Marin, A. FDI spillovers, absorptive capacities and human capital development: evidence from Argentina. MERIT Research Memorandum 2003 $-016,2003$.

Pack, H. The role of exports in asian development. In: Birdsall, N.; Jaspersen, F. (eds.), Pathways to growth: comparing East Asia and Latin America. Washington DC: Inter-American Development Bank, 1997.

Ponomareva, N. Are there positive or negative spillovers from foreign-owned to domestic firms?. Moscovo: New Economic School, 2000. (Working Paper BSP/00/042).

Proença, I.; Fontoura, M.; Crespo, N. Productivity spillovers from multinational corporations in the portuguese case: evidence from a short time period panel data.. Departamento de Economia, CEDIN, CEMAPRE, ISEG/UTL, Universidade Técnica de Lisboa, 2002. (Working Paper 06/2002)

; Productivity spillovers from multinational corporations: vulnerability to deficient estimation. Applied Econometrics and International Development, 6(1), p. 87-98, 2006.

Reis, C. Investimento directo estrangeiro e spillovers sobre a produtividade - um estudo aplicado à indústria transformadora portuguesa. 2001. Dissertação (Mestrado em Economia), Faculdade de Economia, Universidade de Coimbra.

Rhee, Y. The catalyst model of development: lessons from Bangladesh's success with garment exports. World Development, 18(2), p. 333-346, 1990.

Rodríguez-Clare, A. Multinationals, linkages, and economic development. American Economic Review, 86 (4), p. 852-873, 1996.

Ruane, F.; Ugur, A. Foreign direct investment and productivity spillovers in the irish manufacturing industry: evidence from firm level panel data. Trinity College Dublin, 2002. (Trinity Economic Papers 02/06).

Saggi, K. Trade, foreign direct investment, and international technology transfer: a survey. The World Bank Research Observer, 17(2), p. 191-235, 2002.

Santos, V. Investimento directo estrangeiro e a eficiência da indústria portuguesa. Estudos de Economia, 11 (2), p. 181-201, 1991. 
Schoors, K.; van der Tol, B. Foreign direct investment spillovers within and between sectors: evidence from hungarian data. University of Gent, 2002. (Working Paper 2002/157)

Sgard, J. Direct foreign investments and productivity growth in hungarian firms, 1992-1999. William Davidson Institute Working Paper No. 425, 2001.

Sinani, E.; Meyer, K. Spillovers of technology transfer from FDI: the case of Estonia. Journal of Comparative Economics, 32, p. 445-466, 2004.

Sjöholm, F. Technology gap, competition and spillovers from direct foreign investment: evidence from establishment data. Journal of Development Studies, 36(1), p.53-73, 1999a.

; Productivity growth in Indonesia: the role of regional characteristics and direct foreign investment. Economic Development and Cultural Change, 47(3), p. $559-584,1999$ b.

Teece, D. Technology transfer by multinational firms: the resource cost of transferring technological know-how. Economic Journal, 87, p. 242-261, 1977.

Torlak, E. Foreign direct investment, technology transfer, and productivity growth in transition countries - empirical evidence from panel data. Cege Discussion Paper No. 26, 2004.

Wang, J.; Blomström, M. Foreign investment and technology transfer: a simple model. European Economic Review, 36, p. 137 - 155, 1992.

Yudaeva, K.; Kozlov, K.; Malentieva, N.; Ponomareva, N. Does foreign ownership matter? the russian experience. Economics of Transition, 11(3), p. 383-409, 2003. 